\section{International Scientific Journal Theoretical \& Applied Science}

\author{
p-ISSN: 2308-4944 (print) e-ISSN: 2409-0085 (online) \\ Year: $2016 \quad$ Issue: 9 Volume: 41 \\ Published: $30.09 .2016 \quad$ http://T-Science.org
}

SECTION 2. Applied mathematics. Mathematical modeling.

\section{Denis Chemezov}

Master of Engineering and Technology, Corresponding Member of International Academy of Theoretical and Applied Sciences, Lecturer of Vladimir Industrial College, Russian Federation chemezov-da@yandex.ru

Nikita Palev Student of Vladimir Industrial College, Russian Federation nik-palev@yandex.ru

\title{
ANALYTICAL MODELS OF THE TURBULENT FLUID FLOW IN A CIRCULAR PIPE
}

Abstract: The article is discussed the character of the fluid flow (water) on the cylindrical section of the circular pipe. The formulas for calculating the parameters of the vortex flow of a fluid on the basis of the $k$ - $\varepsilon$ turbulence model are presented.

Key words: turbulent flow, formula, $k$-e model, component, pipe, fluid.

Language: Russian

Citation: Chemezov D, Palev N (2016) ANALYTICAL MODELS OF THE TURBULENT FLUID FLOW IN A CIRCULAR PIPE. ISJ Theoretical \& Applied Science, 09 (41): 77-84.

Soi: http://s-o-i.org/1.1/TAS-09-41-12 Doi: crossef http://dx.doi.org/10.15863/TAS.2016.09.41.12

\section{АНАЛИТИЧЕСКИЕ МОДЕЛИ ТУРБУЛЕНТНОГО ТЕЧЕНИЯ ЖИДКОСТИ В КРУГЛОЙ ТРУБЕ}

Аннотация: В статье рассматривается характер течения жидкости (воды) на цилиндрическом участке круглой трубы. Представлены формулы для расчета параметров вихревого течения жидкости на основании $k$ - $\varepsilon$ модели турбулентности.

Ключевые слова: турбулентное течение, формула, $k$-є модель, составляющая, труба, жидкость.

\section{ВВЕДЕНИЕ}

Турбулентное течение характеризуется интенсивным перемешиванием жидкости с изменением величин скоростей и давлений. Описание процесса турбулентного течения жидкости можно представить различными моделями, которые отличаются между собой сложностью и точностью решения [1]. Различают модели турбулентности нулевого уравнения (модель вихревой вязкости во внутренних слоях жидкости; модель Себеси-Смита; модель Болдуина-Ломакса; модель турбулентных струй; модель Буссинеска), с одним уравнением (модель Спаларта-Аллмараса), с двумя уравнениями ( $k-\varepsilon$ модели - стандартная, низкорейнольдсовая и нелинейная; $k$ - $\omega$ модели; модель Ментера; модель RNG) и гибридные модели турбулентности RANS/LES. Модели нулевого уравнения являются наиболее простыми моделями, которые дают явные алгебраические выражения для турбулентной вязкости. Гибридные модели турбулентности RANS/LES представляются осредненными по Рейнольдсу уравнениями Навье-Стокса [2] в условиях моделирования крупных вихрей. Модели турбулентности с двумя уравнениями в полном объеме описывают характер турбулентного течения жидкости при средних объемах моделирования. Рассмотрим одну из моделей турбулентности с двумя уравнениями и дадим математическое описание процесса течения жидкости в круглой трубе.

Компьютерное моделирование процесса турбулентного течения воды в круглой трубе реализовывалось в модуле CFD программного комплекса COMSOL Multiphysics 5.1 [3, 4]. Твердотельная объемная модель фрагмента круглой трубы импортировалась из программной среды SolidWorks посредством модуля LiveLink $^{\text {TM }}$ for SOLIDWORKS ${ }^{\circledR}$. Внутренний диаметр модели стальной трубы принимался 14 мм, длина - 30 мм.

Во вкладке «Физика» были установлены параметры ламинарного течения воды при температуре $T 293.14$ К (однофазный поток). Дискретизация потока жидкости принималась по умолчанию P1+P1 (линейные элементы и компоненты поля давлений).

Моделирование турбулентного течения осуществлялось методом уравнений НавьеСтокса, осреднённых по Рейнольдсу (RANS). 
Данный метод используется для описания турбулентных течений жидкости, при замене случайно изменяющихся характеристик потока суммами осреднённых и пульсационных составляющих.

Расчет производился по $k$ - $\varepsilon$ модели турбулентности для несжимаемого потока жидкости [5]. $k$ - $\varepsilon$ модель относится к классу дифференциальных моделей турбулентности с двумя уравнениями для описания сдвиговой несжимаемой турбулентности.

Параметр сглаживания $\sigma_{w}$ и закон стенки трубы $B$ (для гладких труб) задавались универсальными постоянными величинами 0.2 и
5.2 соответственно. Пористость $\varepsilon_{p}$ для принятой жидкости установлена величиной 1. Давление воды в круглой трубе принималось величиной 50 кПа при коэффициенте изотермической сжимаемости [6] $\beta_{T}$ равном $001 /$ Па. Поле скоростей течения жидкости раскладывается на 3 составляющие (по координатным осям). Для составляющих поля скоростей течения воды были установлены следующие значения: по оси $x$ $=0 \mathrm{M} / \mathrm{c}$, по оси $y=0 \mathrm{M} / \mathrm{c}$ и по оси $z=3 \mathrm{M} / \mathrm{c}$.

Параметры разбиения модели фрагмента круглой трубы на конечные элементы [7] представлены в табл. 1.

\section{Статистика и размеры сетки.}

Таблица 1

\begin{tabular}{|c|c|}
\hline Наименование & Величина \\
\hline Минимальное качество элемента & 0.02121 \\
\hline Среднее качество элемента & 0.5031 \\
\hline Тетраэдральные элементы & 258917 \\
\hline Призматические элементы & 44952 \\
\hline Треугольные элементы & 780 \\
\hline Граничные элементы & 8 \\
\hline Вершины & 0.954 \\
\hline Максимальный размер элемента & 0.18 \\
\hline Минимальный размер элемента & 0.5 \\
\hline Коэффициент кривизны & 0.8 \\
\hline Минимальное количество элементов по самой короткой границе & 1.13 \\
\hline Максимальный темп роста элемента & \\
\hline
\end{tabular}

Запишем уравнение количества движения для несжимаемого потока жидкости при незначительном изменении величины плотности и температуры (1)

$$
\rho(u \cdot \nabla) \cdot u=\nabla \cdot\left[-p I+\left(\mu+\mu_{t}\right)\left(\nabla u+(\nabla u)^{T}\right)\right]+F,
$$

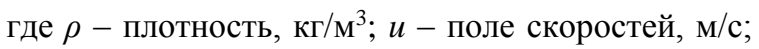
$\nabla$ - оператор Гамильтона; $p$ - давление, Па; $I$ единичный тензор; $\mu$ - динамическая вязкость, Па · c; $\mu_{t}$ - турбулентная динамическая вязкость, Па · с; $\nabla u$ - градиент $u$, м/с; $T-$ абсолютная температура, К; $F$ - объемная сила, Н/ ${ }^{3}$.

Турбулентная динамическая вязкость $\mu_{t}$ определяется по формуле (2)

$$
\mu_{t}=\rho \cdot C_{\mu} \frac{k^{2}}{\varepsilon}
$$

где $C_{\mu}$ - параметр $k$ - $\varepsilon$ модели турбулентности (константа) [8], $C_{\mu}=0.09 ; k$-кинетическая энергия турбулентности, $\mathrm{M}^{2} / \mathrm{c}^{2} ; \varepsilon-$ скорость вязкой диссипации кинетической энергии турбулентности, $\mathrm{M}^{2} / \mathrm{c}^{3}$.

При этом должно выполняться условие (3)

$$
\rho \nabla \cdot(u)=0
$$

Для $k$ - $\varepsilon$ модели турбулентности запишем уравнение (первое) баланса кинетической энергии турбулентности (4). $k$ - это энергия соответствующая пульсационным скоростям турбулентного движения в жидкости и отнесенная к ее массе.

$$
\rho(u \cdot \nabla) \cdot k=\nabla \cdot\left[\left(\mu+\frac{\mu_{t}}{\sigma_{k}}\right) \nabla k\right]+P_{k}-\rho \varepsilon,
$$

где $\sigma_{k}-$ параметр $k$ - $\varepsilon$ модели турбулентности (константа), $\sigma_{k}=1.0 ; \nabla k$ - градиент $k, \mathrm{M}^{2} / \mathrm{c}^{2} ; P_{k}-$ генерация кинетической энергии турбулентности, $\mathrm{BT} / \mathrm{M}^{3}$. 


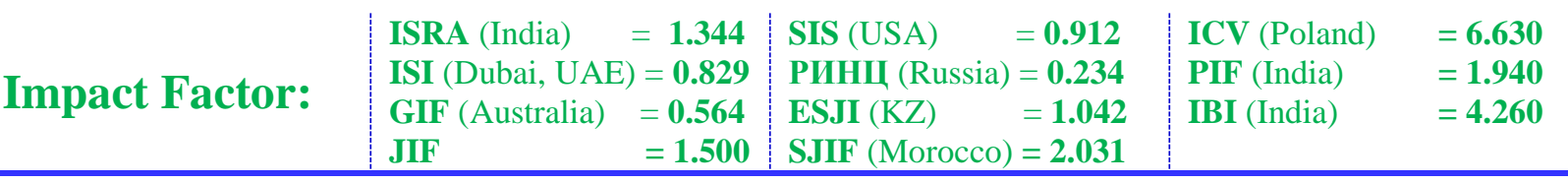

Генерация кинетической энергии турбулентности $P_{k}$ определяется по формуле (5)

$$
P_{k}=\mu_{t}\left[\nabla u:\left(\nabla u+(\nabla u)^{T}\right)\right]
$$

где : - оператор «отношение между градиентами». Запишем уравнение (второе) баланса скорости вязкой диссипации кинетической энергии турбулентности (6). $\varepsilon-$ скорость, с которой $k$ превращается в тепло вследствие вязкого трения.

$$
\rho(u \cdot \nabla) \cdot \varepsilon=\nabla \cdot\left[\left(\mu+\frac{\mu_{t}}{\sigma_{\varepsilon}}\right) \nabla \varepsilon\right]+C_{\varepsilon 1} \frac{\varepsilon}{k} P_{k}-C_{\varepsilon 2} \rho \frac{\varepsilon^{2}}{k}
$$

где $\sigma_{\varepsilon}-$ параметр $k$ - $\varepsilon$ модели турбулентности (константа), $\sigma_{\varepsilon}=1.3 ; \nabla \varepsilon-$ градиент $\varepsilon, \mathrm{M}^{2} / \mathrm{c}^{3} ; C_{\varepsilon l}-$ параметр $k-\varepsilon$ модели турбулентности (константа), $C_{\varepsilon 1}=1.44 ; \quad C_{\varepsilon 2}-$ параметр $k-\varepsilon$ модели турбулентности (константа), $C_{\varepsilon 2}=1.92$. Граничные условия для стенки круглой трубы представлены в формулах (7), (8) и (9)

$$
u \cdot n=0,
$$

где $n$ - нормаль к границе (вектор всегда направлен внутрь расчетной области). Жидкость скользит по внутренней стенке круглой трубы. Скорость течения воды у стенки трубы равна нулю.

$$
\begin{gathered}
\nabla k \cdot n=0 \\
{\left[\left(\mu+\mu_{t}\right)\left(\nabla u+(\nabla u)^{T}\right)\right] \cdot n=-\rho \frac{u_{\tau}}{\delta_{w}^{+}} u_{\text {tan } g .},}
\end{gathered}
$$

где $u_{\tau}$ - динамическая (сдвиговая) скорость, м/с; $\delta_{w}^{+}-$расстояние от внутренней поверхности круглой трубы до границы течения жидкости с учетом вязкости, мм; $u_{\text {tang. }}$ - тангенциальная скорость, м/с.

$u_{\text {tang. }}$ определяется по формуле (10)

$$
u_{\text {tan } g .}=u-(u \cdot n) \cdot n
$$

Для развитых турбулентных потоков скорость вязкой диссипации кинетической энергии турбулентности $\varepsilon$ определяется по формуле (11)

$$
\varepsilon=\rho \frac{C_{\mu} k^{2}}{k_{v} \delta_{w}^{+} \mu}
$$

где $k_{v}$ - параметр $k$ - $\varepsilon$ модели турбулентности (константа), $k_{v}=0.41$.

Величина абсолютного давления $p_{a b s .}$ жидкости определяется по формуле (12)

$$
p_{\text {abs. }}=p+p_{\text {ref. }},
$$

где $p_{r e f .}-$ стандартное атмосферное давление, $p_{r e f}$. $=101.325$ кПа.

Упорядоченная кинетическая энергия турбулентности потока жидкости $k_{\text {reg. }}\left(\mathrm{M}^{2} / \mathrm{c}^{2}\right)$ равна максимальному значению двух аргументов.

$$
k_{\text {reg. }}=\max (k, 0)
$$

Упорядоченная скорость вязкой диссипации кинетической энергии турбулентности потока жидкости $\varepsilon_{\text {reg. }}\left(\mathrm{M}^{2} / \mathrm{c}^{3}\right)$ равна максимальному значению двух аргументов.

$$
\varepsilon_{\text {reg. }}=\max (\varepsilon, 0)
$$

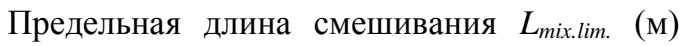
жидкости на цилиндрическом участке круглой трубы при турбулентном режиме определяется по формуле (15)

$$
L_{\text {mix.lim. }}=2 \cdot L_{\text {ref. }},
$$

где $L_{r e f .}-$ базовая длина, $L_{\text {ref. }}=0.008999999999999994 \mathrm{M}$.

По формулам (16) и (17) находят расчетные кинетическую энергию турбулентности $k_{\text {cal }}$. $\left(\mathrm{M}^{2} / \mathrm{c}^{2}\right)$ и скорость вязкой диссипации кинетической энергии турбулентности $\varepsilon_{c a l .}\left(\mathrm{m}^{2} / \mathrm{c}^{3}\right)$.

$$
\begin{aligned}
& k_{\text {cal. }}=\left[\frac{100 \mu}{\rho \cdot L_{\text {mix.lim. }}}\right]^{2} \\
& \varepsilon_{\text {cal. }}=\frac{10 \cdot C_{\mu} \cdot \sqrt{k^{3}}}{L_{\text {mix.lim. }}}
\end{aligned}
$$

$k$-уравнение, линейный коэффициент $C_{k}$ (кг/(м ${ }^{3}$. c)) будет равен произведению двух переменных $\rho$ и $\gamma_{t}$ (18). Плотность воды в формуле отрицательное значение.

$$
C_{k}=-\rho \cdot \gamma_{t}
$$

где $\gamma_{t}$ - вспомогательная переменная, (1/c).

Составляющие скорости деформации $S$ представлены в системе уравнений (19) 


\begin{tabular}{|c|c|c|c|c|c|c|}
\hline Impact Factor: & $\begin{array}{l}\text { ISRA (India) } \\
\text { ISI (Dubai, UAE } \\
\text { GIF (Australia) } \\
\text { JIF }\end{array}$ & $\begin{array}{l}=1.344 \\
=0.829 \\
=0.564 \\
=1.500\end{array}$ & $\begin{array}{l}\text { SIS (USA) } \\
\text { PИНЦ (Russia) } \\
\text { ESJI (KZ) } \\
\text { SJIF (Morocco) }\end{array}$ & $\begin{array}{l}=0.912 \\
=0.234 \\
=1.042 \\
=2.031\end{array}$ & $\begin{array}{l}\text { ICV (Poland) } \\
\text { PIF (India) } \\
\text { IBI (India) }\end{array}$ & $\begin{array}{l}=6.630 \\
=1.940 \\
=4.260\end{array}$ \\
\hline
\end{tabular}

$$
\left\{\begin{array}{l}
S_{x x}=u x \\
S_{y x}=0.5(v x+u y) \\
S_{z x}=0.5(w x+u z) \\
S_{x y}=0.5(u y+v x) \\
S_{y y}=v y \\
S_{z y}=0.5(w y+v z) \\
S_{x z}=0.5(u z+w x) \\
S_{y z}=0.5(v z+w y) \\
S_{z z}=w z
\end{array},\right.
$$

где $S$ - скорость деформации, $1 / \mathrm{c} ; x x, y x, z x, x y, y y$, $z y, x z, y z, z z$ - составляющие; $u, v, w$ - проекции вектора скорости соответственно на оси $x, y, z$.

$$
S_{a b s}=\sqrt{\left(S_{x x}\right)^{2}+\left(S_{x y}\right)^{2}+\left(S_{x z}\right)^{2}+\left(S_{y x}\right)^{2}+\left(S_{y y}\right)^{2}+\left(S_{y z}\right)^{2}+\left(S_{z x}\right)^{2}+\left(S_{z y}\right)^{2}+\left(S_{z z}\right)^{2}}
$$

Источник действия кинетической энергии турбулентности $k_{s}\left(1 / \mathrm{c}^{2}\right)$ и скорость сдвига $e(1 / \mathrm{c})$ движущихся слоев жидкости в круглой трубе определяются по формулам (21) и (22)

$$
\begin{gathered}
k_{s}=2 \cdot(u x)^{2}+u y(u y+v x)+u z(u z+w x)+v x(u y+v x)+2 \cdot(v y)^{2}+v z(v z+w y)+w x(u z+w x)+w y(v z+w y)+2 \cdot(w z)^{2} \\
e=\sqrt{0.5\left(4 \cdot(u x)^{2}+2 \cdot(u y+v x)^{2}+2 \cdot(u z+w x)^{2}+4 \cdot(v y)^{2}+2 \cdot(v z+w y)^{2}+4 \cdot(w z)^{2}\right)+e p s}
\end{gathered}
$$

Дивергенция [9] div u (1/c) равна сумме полей скоростей турбулентного течения жидкости действующих по координатным осям (23)

$$
\operatorname{div} u=u x+v y+w z
$$

Величина скорости потока жидкости $U$ (м/с) в турбулентном режиме определяется по формуле (24). При турбулентном движении (неустановившемся) жидкости с заданными граничными условиями, величины осреднённых скоростей меняются во времени.

$$
U=\sqrt{u^{2}+v^{2}+w^{2}}
$$

Составляющие вихревого поля потока жидкости $\Omega(1 / \mathrm{c})[10]$ в турбулентном режиме представлены в системе, состоящей из трех уравнений (25)

$$
\left\{\begin{array}{l}
\Omega_{x}=w y-v z \\
\Omega_{y}=-w x+u z \\
\Omega_{z}=v x-u y
\end{array}\right.
$$

где $\Omega_{x}, \Omega_{y}, \Omega_{z}$ - составляющие вихревого поля потока $\Omega$.

Величина $\Omega$ равна

$$
\Omega=\sqrt{\Omega_{x}^{2}+\Omega_{y}^{2}+\Omega_{z}^{2}}
$$

Величина кинематической вязкости $v\left(\mathrm{M}^{2} / \mathrm{c}\right)$ жидкости равна отношению $\mu$ к $\rho$. Вязкость характеризует степень текучести воды и подвижности ее частиц.

$$
v=\frac{\mu}{\rho}
$$

Вязкостное напряжение - сила вязкости, приходящаяся на единицу площади поверхности раздела двух слоев. Составляющие вязкостного напряжения $\sigma_{\eta}\left(\mathrm{H} / \mathrm{M}^{2}\right)$ находятся по формулам (28)

$\left\{\begin{array}{l}\sigma_{\eta x}=\mu \cdot\left(2 \cdot u x \cdot n_{x m e s h}+(u y+v x) \cdot n_{y m e s h}+(u z+w x) \cdot n_{z m e s h}\right) \\ \sigma_{\eta y}=\mu \cdot\left((v x+u y) \cdot n_{x m e s h}+2 \cdot v y \cdot n_{y m e s h}+(v z+w y) \cdot n_{z m e s h}\right), \\ \sigma_{\eta z}=\mu \cdot\left((w x+u z) \cdot n_{x m e s h}+(w y+v z) \cdot n_{y m e s h}+2 \cdot w z \cdot n_{z m e s h}\right)\end{array}\right.$

где $n_{x m e s h}, n_{y m e s h}, \quad n_{z m e s h}-$ составляющие нормального вектора.

Составляющие полного напряжения $\sigma_{t o t}$ $\left(\mathrm{H} / \mathrm{M}^{2}\right)$ представлены в системе уравнений (29)

$$
\left\{\begin{array}{l}
\sigma_{t o t x}=\sigma_{\eta x}-p \cdot n_{x m e s h} \\
\sigma_{t o t y}=\sigma_{\eta y}-p \cdot n_{y m e s h} \\
\sigma_{t o t z}=\sigma_{\eta z}-p \cdot n_{z m e s h}
\end{array}\right.
$$




\begin{tabular}{|c|c|c|c|c|c|c|}
\hline Impact Factor: & $\begin{array}{l}\text { ISRA (India) } \\
\text { ISI (Dubai, UAE } \\
\text { GIF (Australia) } \\
\text { JIF }\end{array}$ & $\begin{array}{l}=1.344 \\
=0.829 \\
=0.564 \\
=1.500\end{array}$ & $\begin{array}{l}\text { SIS (USA) } \\
\text { PИНЦ (Russia) } \\
\text { ESJI (KZ) } \\
\text { SJIF (Morocco) }\end{array}$ & $\begin{array}{l}=0.912 \\
=0.234 \\
=1.042 \\
=2.031\end{array}$ & $\begin{array}{l}\text { ICV (Poland) } \\
\text { PIF (India) } \\
\text { IBI (India) }\end{array}$ & $\begin{array}{l}=6.630 \\
=1.940 \\
=4.260\end{array}$ \\
\hline
\end{tabular}

где $\sigma_{\text {totx }}, \sigma_{\text {toty }}, \sigma_{\text {totz }}-$ составляющие полного напряжения $\sigma_{\text {tot. }}$.
Составляющие скорости напряжения сдвига $\tau_{v d}$ (Па) слоев жидкости определяются по формулам (30)

$$
\left\{\begin{array}{l}
\tau_{v d x x}=2 \cdot \mu \cdot u x \\
\tau_{v d y x}=\mu \cdot(v x+u y) \\
\tau_{v d z x}=\mu \cdot(w x+u z) \\
\tau_{v d x y}=\mu \cdot(u y+v x) \\
\tau_{v d y y}=2 \cdot \mu \cdot v y \\
\tau_{v d z y}=\mu \cdot(w y+v z) \\
\tau_{v d x z}=\mu \cdot(u z+w x) \\
\tau_{v d y z}=\mu \cdot(v z+w y) \\
\tau_{v d z z}=2 \cdot \mu \cdot w z
\end{array},\right.
$$

где $\tau_{v d x x}, \tau_{v d y x}, \tau_{v d z x}, \tau_{v d x y}, \tau_{v d y y}, \tau_{v d z y}, \tau_{v d x z}, \tau_{v d y z}, \tau_{v d z z}-$ составляющие скорости напряжения сдвига $\tau_{v d}$.

Вязкое рассеяние или вязкостная диссипация (преобразование кинетической энергии во внутреннюю энергию) $Q_{\eta}\left(\mathrm{BT} / \mathrm{M}^{3}\right)$ рассчитывается по формуле (31)

$$
Q_{\eta}=\tau_{v d x x} \cdot u x+\tau_{v d x y} \cdot u y+\tau_{v d x z} \cdot u z+\tau_{v d y x} \cdot v x+\tau_{v d y y} \cdot v y+\tau_{v d y z} \cdot v z+\tau_{v d z x} \cdot w x+\tau_{v d z y} \cdot w y+\tau_{v d z z} \cdot w z+\rho+\varepsilon_{r e g}
$$

Разностные уравнения для кинетической энергии турбулентности $k_{\text {res. }}\left(\mathrm{BT} / \mathrm{M}^{3}\right)$ и скорости турбулентности $\varepsilon_{\text {res. }}\left(\Pi \mathrm{a} / \mathrm{c}^{2}\right)$ будут иметь вязкой диссипации кинетической энергии следующий вид (32) и (33)

$$
\begin{gathered}
k_{\text {res. }}=-\left(\mu+\frac{\mu_{t}}{\sigma_{k}}\right) \cdot\left(k_{x x}+k_{y y}+k_{z z}\right)-C_{k} \cdot k-P_{k}+\rho \cdot u \cdot k_{x}+\rho \cdot v \cdot k_{y}+\rho \cdot w \cdot k_{z} \\
\varepsilon_{\text {res. }}=-\left(\mu+\frac{\mu_{t}}{\sigma_{\varepsilon}}\right) \cdot\left(\varepsilon_{x x}+\varepsilon_{y y}+\varepsilon_{z z}\right)-C_{\text {slin. }} \cdot \varepsilon-P_{\varepsilon}+\rho \cdot u \cdot \varepsilon_{x}+\rho \cdot v \cdot \varepsilon_{y}+\rho \cdot w \cdot \varepsilon_{z},
\end{gathered}
$$

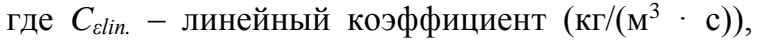
определяется по формуле (34). $P_{\varepsilon}$ равен максимальному значению двух приведенных аргументов.

$$
\begin{gathered}
C_{\text {gin }}=-\rho \cdot C_{\varepsilon 2} \cdot y_{t} \\
P_{\varepsilon}=\max \left(C_{\varepsilon 1} \cdot y_{t} \cdot \mu_{t} \cdot k_{s}, 0\right)
\end{gathered}
$$

Разностное уравнение для поля скоростей $u_{\text {res. }}\left(\mathrm{H} / \mathrm{M}^{3}\right)$ турбулентного течения жидкости с учетом действия объемной силы будет иметь следующий вид

$$
u_{\text {res. }}=p x+\rho \cdot u \cdot u x+\rho \cdot v \cdot u y+\rho \cdot w \cdot u z-\left(\frac{\partial(2 \cdot u x)}{\partial x}+\frac{\partial(u y+v x)}{\partial y}+\frac{\partial(u z+w x)}{\partial z}\right) \cdot\left(\mu+\mu_{t}\right)-F_{x},
$$

где $F_{x}$ - составляющая объемной силы $\left(\mathrm{H} / \mathrm{M}^{3}\right)$.

Разностное уравнение для поля скоростей

учетом действия объемной силы будет иметь следующий вид

$v_{\text {res. }}\left(\mathrm{H} / \mathrm{M}^{3}\right)$ турбулентного течения жидкости с

$$
v_{r e s .}=\rho \cdot u \cdot v x+p y+\rho \cdot v \cdot v y+\rho \cdot w \cdot v z-\left(\frac{\partial(v x+u y)}{\partial x}+\frac{\partial(2 \cdot v y)}{\partial y}+\frac{\partial(v z+w y)}{\partial z}\right) \cdot\left(\mu+\mu_{t}\right)-F_{y},
$$

где $F_{y}$ - составляющая объемной силы $\left(\mathrm{H} / \mathrm{M}^{3}\right)$.

Разностное уравнение для поля скоростей $w_{\text {res. }}\left(\mathrm{H} / \mathrm{M}^{3}\right)$ турбулентного течения жидкости с учетом действия объемной силы будет иметь следующий вид 


\begin{tabular}{|c|c|c|c|c|c|c|}
\hline Impact Factor: & $\begin{array}{l}\text { ISRA (India) } \\
\text { ISI (Dubai, UAE } \\
\text { GIF (Australia) } \\
\text { JIF }\end{array}$ & $\begin{array}{l}=1.344 \\
=0.829 \\
=0.564 \\
=1.500\end{array}$ & $\begin{array}{l}\text { SIS (USA) } \\
\text { PИНЦ (Russia) } \\
\text { ESJI (KZ) } \\
\text { SJIF (Morocco) }\end{array}$ & $\begin{array}{l}=0.912 \\
=0.234 \\
=1.042 \\
=2.031\end{array}$ & $\begin{array}{l}\text { ICV (Poland) } \\
\text { PIF (India) } \\
\text { IBI (India) }\end{array}$ & $\begin{array}{l}=6.630 \\
=1.940 \\
=4.260\end{array}$ \\
\hline
\end{tabular}

$$
w_{r e s .}=\rho \cdot u \cdot w x+\rho \cdot v \cdot w y+p z+\rho \cdot w \cdot w z-\left(\frac{\partial(w x+u z)}{\partial x}+\frac{\partial(w y+v z)}{\partial y}+\frac{\partial(2 \cdot w z)}{\partial z}\right) \cdot\left(\mu+\mu_{t}\right)-F_{z},
$$

где $F_{z}$ - составляющая объемной силы $\left(\mathrm{H} / \mathrm{M}^{3}\right)$.

Разностное уравнение для давления $p_{\text {res. }}$

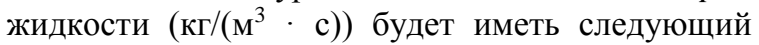
вид

$$
p_{\text {res. }}=\rho \cdot \operatorname{div} u
$$

Слабая форма аналитически выводится для моделей, включающих сложные граничные условия с зависимостями от переменных, системы отсчета, времени. Слабая форма для $k$ будет иметь следующий вид

$$
\begin{gathered}
-\operatorname{test}\left(k_{x}\right) \cdot\left(\mu+\frac{\mu_{t}}{\sigma_{k}}\right) \cdot k_{x}-\operatorname{test}\left(k_{y}\right) \cdot\left(\mu+\frac{\mu_{t}}{\sigma_{k}}\right) \cdot k_{y}-\operatorname{test}\left(k_{z}\right) \cdot\left(\mu+\frac{\mu_{t}}{\sigma_{k}}\right) \cdot k_{z}-\rho \cdot\left(u \cdot k_{x}+v \cdot k_{y}+w \cdot k_{z}\right), \\
\cdot \operatorname{test}(k)+P_{k} \cdot \operatorname{test}(k)+C_{k} \cdot k \cdot \operatorname{test}(k)
\end{gathered}
$$

где - test специальный оператор, создает тестовую функцию для переменной.

Слабая форма для $\varepsilon$ будет иметь следующий вид

$$
\begin{gathered}
-\operatorname{test}\left(\varepsilon_{x}\right) \cdot\left(\mu+\frac{\mu_{t}}{\sigma_{\varepsilon}}\right) \cdot \varepsilon_{x}-\operatorname{test}\left(\varepsilon_{y}\right) \cdot\left(\mu+\frac{\mu_{t}}{\sigma_{\varepsilon}}\right) \cdot \varepsilon_{y}-\operatorname{test}\left(\varepsilon_{z}\right) \cdot\left(\mu+\frac{\mu_{t}}{\sigma_{\varepsilon}}\right) \cdot \varepsilon_{z}-\rho \cdot\left(u \cdot \varepsilon_{x}+v \cdot \varepsilon_{y}+w \cdot \varepsilon_{z}\right) \cdot \\
\cdot \operatorname{test}(\varepsilon)+P_{\varepsilon} \cdot \operatorname{test}(\varepsilon)+C_{\text {sin }} \cdot \varepsilon \cdot \operatorname{test}(\varepsilon)
\end{gathered}
$$

Слабые формы для объемной силы, действующей на внутреннюю поверхность трубы и для полей скоростей турбулентного течения жидкости, представлены формулами (42) и (43) соответственно

$$
\rho \cdot\left[\begin{array}{c}
-\left(\frac{\partial(u, x)}{\partial x} \cdot u+\frac{\partial(u, y)}{\partial y} \cdot v+\frac{\partial(u, z)}{\partial z} \cdot w\right) \cdot \operatorname{test}(u)-\left(\frac{\partial(v, x)}{\partial x} \cdot u+\frac{\partial(v, y)}{\partial y} \cdot v+\frac{\partial(v, z)}{\partial z} \cdot w\right) \cdot \\
\cdot \operatorname{test}(v)-\left(\frac{\partial(w, x)}{\partial x} \cdot u+\frac{\partial(w, y)}{\partial y} \cdot v+\frac{\partial(w, z)}{\partial z} \cdot w\right) \cdot \operatorname{test}(w)
\end{array}\right]
$$

С помощью функций формы выполняется аппроксимация поля неизвестных величин. Функция формы для $\varepsilon$ будет иметь следующий вид

$$
-\varepsilon+\varepsilon_{w}
$$

Ход расчета величин $u, p, k$ и $\varepsilon$ представлен сегрегированными шагами 1 и 2 (первая и седьмая итерации). Решатель - PARDISO (Parallel Direct Sparse Solver for Clusters), работает c общими системами $A x=B$ и использует методику волнения центра, которая проверяет величину потенциального центра относительно постоянного некоторого порога, что позволяет учитывать особые точки и компенсировать их.

Segregated solver

Number of degrees of freedom solved for: 1203246.

Segregated solver iteration 1.

Segregated Step 1

Nonsymmetric matrix found.

Scales for dependent variables:

Pressure (comp1.p): 5e+004

Velocity field (comp1.u): $6 \mathrm{e}+002$

Orthonormal null-space function used.

ISPC Global Applied Research, 


\begin{tabular}{|c|c|c|c|c|c|c|}
\hline Impact Factor: & $\begin{array}{l}\text { ISRA (India) } \\
\text { ISI (Dubai, UAE } \\
\text { GIF (Australia) } \\
\text { JIF }\end{array}$ & $\begin{array}{l}=1.344 \\
=0.829 \\
=0.564 \\
=1.500\end{array}$ & $\begin{array}{l}\text { SIS (USA) } \\
\text { PИНЦ (Russia) } \\
\text { ESJI (KZ) } \\
\text { SJIF (Morocco) }\end{array}$ & $\begin{array}{l}=0.912 \\
=0.234 \\
=1.042 \\
=2.031\end{array}$ & $\begin{array}{l}\text { ICV (Poland) } \\
\text { PIF (India) } \\
\text { IBI (India) }\end{array}$ & $\begin{array}{l}=6.630 \\
=1.940 \\
=4.260\end{array}$ \\
\hline
\end{tabular}

Iter SolEst Damping Stepsize \#Res \#Jac \#Sol LinIt LinErr LinRes

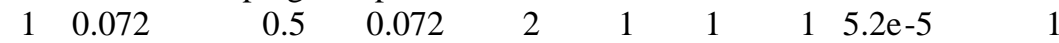

Segregated Step 2

Nonsymmetric matrix found.

Scales for dependent variables:

Turbulent dissipation rate (comp1.ep): 3.2e-005

Turbulent kinetic energy (comp1.k): 3.1e-005

Orthonormal null-space function used.

Iter SolEst ResEst Damping Stepsize \#Res \#Jac \#Sol LinIt LinErr LinRes

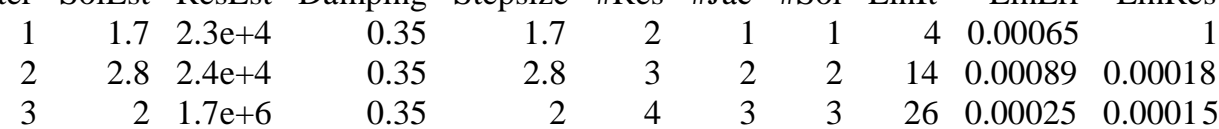

Solution error estimates for segregated groups

$0.036,1.8$

Residual error estimates for segregated groups

$1.7 \mathrm{e}+004,3.5 \mathrm{e}+004$

Segregated solver iteration 7.

Segregated Step 1

Iter SolEst Damping Stepsize \#Res \#Jac \#Sol LinIt LinErr LinRes

$\begin{array}{llllllllll}1 & 3.7 & 0.5 & 3.7 & 14 & 7 & 7 & 111 & 0.00072 & 0.00031\end{array}$

Segregated Step 2

Scales for dependent variables:

Turbulent dissipation rate (comp1.ep): 51

Turbulent kinetic energy (comp1.k): 0.034

Iter SolEst ResEst Damping Stepsize \#Res \#Jac \#Sol LinIt LinErr LinRes

$\begin{array}{rrrrrrrrrrr}1 & 1.1 & 8.3 \mathrm{e}+3 & 0.35 & 1.1 & 26 & 19 & 19 & 133 & 0.00016 & 2 \mathrm{e}-7 \\ 2 & 0.84 & 1 \mathrm{e}+4 & 0.35 & 0.84 & 27 & 20 & 20 & 136 & 0.00093 & 1.1 \mathrm{e}-6 \\ 3 & 0.58 & 1.6 \mathrm{e}+4 & 0.35 & 0.58 & 28 & 21 & 21 & 139 & 0.00067 & 9.1 \mathrm{e}-7\end{array}$

Solution error estimates for segregated groups

$1.9,0.73$

Residual error estimates for segregated groups

$2.8 \mathrm{e}+003,2.4 \mathrm{e}+008$

\section{ЗАКЛЮЧЕНИЕ}

Модель процесса турбулентного течения жидкости можно представить в виде законов изменения величины поля скоростей потока на рассматриваемой длине круглой трубы. Это изменение сопровождается увеличением значений $k$ и $\varepsilon$ в зависимости от свойств жидкости и, в частности, динамической вязкости. Приведенные аналитические модели турбулентного течения в полной мере определяют характер деформации и напряжения движущихся слоев воды на цилиндрическом участке круглой трубы.

\section{References:}

1. Nichols RH (2010) Turbulence Models and Their Application to Complex Flows. University of Alabama at Birmingham.

2. (2016) Reynolds-averaged Navier-Stokes equations. Available: http://dictionary.sensagent.com/Reynoldsaveraged $\% 20$ Navier-
Stokes\%20equations/en-en/ 20.09.2016).

3. (2011) CFD Module User's Guide, 2011. $444 \mathrm{p}$.

4. Hoffmann KA (2000) Computational fluid dynamics. Wichita: Engineering System. $188 \mathrm{p}$. 


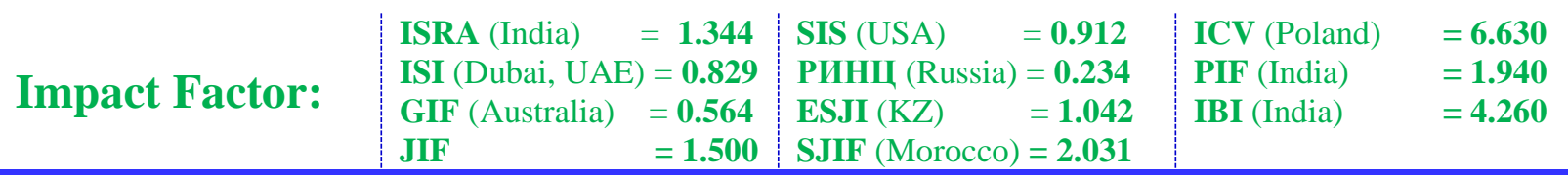

5. Korkodinov IA (2013) The review of set of $\mathrm{k}-\varepsilon$ models for modeling turbulence. Bulletin PNRPU. Mechanical engineering, materials science, №2 (15). - pp. 5- 16 .

6. Kiselev VD, Bolotov AV, Satonin AP, Kashaeva HA, Konovalov AI (2008) Compressibility and Internal Pressure of Liquid. Uchenye Zapiski Kazanskogo Universiteta. Seriya Estestvennye Nauki, vol. 150 . - pp. $76-90$.

7. (2016) Size Parameters for Free Tetrahedral Meshing in COMSOL Multiphysics. Available:

https://www.comsol.it/blogs/sizeparameters-free-tetrahedral-meshing- comsol-multiphysics/

(Accessed: 20.09.2016).

8. Miheev NI, Saushin II (2013) Method and results the estimation of the parameters of the model of turbulence $\mathrm{k}-\varepsilon$ based on experimental velocities fields. Transactions of Academenergo, №3. - pp. 17 - 25.

9. (2016) Divergence. Available: https://en.wikipedia.org/wiki/Divergence (Accessed: 20.09.2016).

10. (2016) Vorticity. Available: https://en.wikipedia.org/wiki/Vorticity (Accessed: 20.09.2016). 